\title{
Estimation of the Carrier-to-Interference Ratio in Cellular Radio Systems
}

\author{
Fredrik Gunnarsson, Jonas Blom and Fredrik Gustafsson \\ Department of Electrical Engineering \\ Linköpings universitet, SE-581 83 Linköping, Sweden \\ WWW: http://wWw.control.isy.liu.se \\ Email: fred@isy.liu.se, jb@isy.liu.se, \\ fredrik@isy.liu.se
}

April 16, 1999

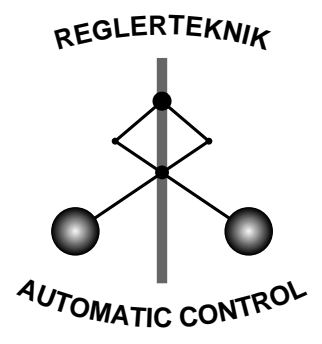

LINKÖPING

Report no.: LiTH-ISY-R-2146

Submitted to VTC'99, Houston, TX, USA

Technical reports from the Automatic Control group in Linkping are available by anonymous ftp at the address ftp.control.isy.liu.se. This report is contained in the compressed postscript file $2146 . \mathrm{ps} . \mathrm{Z}$. 



\begin{abstract}
The problem to track time-varying parameters in cellular radio systems is studied. The focus is on estimation based only on the signals that are readily available. Previous work have demonstrated very good performance relying on analog measurement. In a real system most of the information is lost due to quantization and sampling at a rate that might be as low as $2 \mathrm{~Hz}$ (GSM case). Therefore a different approach is required and for that matter a Maximum Likelihood Estimator has been designed and exemplified in the case of GSM. The needed probability functions of the measurements cannot be described analytically. Instead point-mass approximations can be obtained from Monte-Carlo simulations for each point in a grid covering the interesting parameter space. The proposed algorithm can be tuned to track both slowly and fastly varying parameters individually. Since most computations take place in the base stations, the estimator is ready for implementation in a second generation wireless system. No update of the software in the mobile stations is needed.
\end{abstract}

Keywords: Cellular radio systems; GSM; Maximum Likelihood Estimation; Point-mass approximation; Measurement reports 


\section{Introduction}

For proper operation of a high-capacity cellular radio system, power control is an essential feature. Among others, there are three important aspects to consider.

- Extracting relevant information from the available measurements.

- Design a linear power control algorithm and tune the parameter for optimized performance.

- Incorporate nonlinear components to handle constraints and priorities.

The first issue is dealt with here, while the others are discussed in $[7,6]$. Several transmitter power control algorithms have been proposed to improve the capacity. To avoid extensive signaling in the network, it is desirable to use distributed algorithms, where the transmitter powers are locally controlled based on local measurements or estimates. For an overview, see $[3,4]$ and the references therein. Most algorithms are relying on accurate $\mathrm{C} / \mathrm{I}$ estimates, and popular methods for estimation with good performance are described in $[2,13]$. In those approaches, however, it is assumed that analog signal strength measurements are available, which is not the case in a real system. Instead the information is regularly available in measurement reports. One of the core problems is to locally extract as much relevant information as possible from these reports. This includes the carrier signal and the distribution of the interference, which are used to compute the $\mathrm{C} / \mathrm{I}$. A power control algorithm that is utilizing this additional information is described in [6].

It is common that the measurement reports are comprising coarsely quantized values reflecting the perceived quality (Quality Indicator, QI) and signal strength (Received Signal Strength Indicator, RSSI). These values are depending on the parameters to be estimated. Hence, the measurement reports contain information from two conceptually different information sources, which leads to a sensor fusion formulation of the problem.

This paper utilizes Maximum Likelihood Estimation based on point-mass approximations of the required probability density functions. The system and channel model is described in Section II, followed by a discussion on ML estimation and implementation aspects. The performance is illustrated by simulations, and some concluding remarks are found in Section V.

\section{Modeling}

\subsection{System Model}

Signal gains and power levels can be expressed using either logarithmic (e.g. $d B$ or $d B m$ ) or linear scales. To avoid confusion we will employ the convention of indicating linearly scaled values with a bar. Thus $\bar{g}_{i j}$ is a value in linear scale, and $g_{i j}$ the corresponding value in logarithmic scale. 
Assume that the $m$ mobile stations on a specific radio channel are transmitting using the powers $p_{i}(t)$, where $i=1, \ldots, m$. The signal between mobile station $i$ and base station $j$ is attenuated by the signal gain $g_{i j}(t)(<0)$. Thus the corresponding connected base station will experience a desired carrier signal $C_{i}(t)=p_{i}(t)+g_{i i}(t)$ and an interference plus noise $I_{i}(t)$

$$
I_{i}(t)=10 \log _{10}\left(\sum_{j \neq i} \bar{g}_{i j}(t) \bar{p}_{j}(t)+\bar{\nu}_{i}(t)\right),
$$

where $\bar{\nu}_{i}(t)$ denotes the thermal noise. The $\mathrm{C} / \mathrm{I}$ at base station $i$ is defined by

$$
\gamma_{i}(t)=p_{i}(t)+g_{i i}(t)-I_{i}(t) .
$$

In the rest of the article, only the situation at receiver $i$ will be considered. Therefore, the $i$ index will be dropped for simplicity.

\subsection{Interference}

The interference can be viewed as a stochastic variable with a distribution parameterized by

$$
m_{I}, \sigma_{1}, \ldots, \sigma_{n},
$$

where $m_{I}$ is the mean value. Estimators used in previous work are only characterizing the interference by its mean value, see e.g. [1], and therefore this approach describes the interference more thoroughly. Thus the parameters to be estimated are given by

$$
\boldsymbol{\theta}=\left[C, m_{I}, \sigma_{1}, \ldots, \sigma_{n}\right]^{T} .
$$

As an example, consider frequency hopping GSM, where the characteristics of the interference distribution is approximated by using a simulation model. The gains of the transmitted powers in the random frequency hopping network were modeled by the path loss, shadow fading and multipath fading. Thermal noise was also included in the model. The results are found in Figure 1, from which we conclude that it is reasonable to model the interference as Gaussian. This result proved to be relatively independent not only of network specific parameters such as cell radius and reuse, but also of the distribution of the transmitted powers. Most probably, there are more accurate models, but the issue is essentially to find a model that is good enough to serve the purpose of modeling the interference for estimation. The conclusion is that the interference distribution can approximately be characterized by its mean value $m_{I}$ and its standard deviation $\sigma_{I}$ (different for each user). This is a result in the same direction as in [10]. Thus the parameters to be estimated in this case are

$$
\boldsymbol{\theta}=\left[C, m_{I}, \sigma_{I}\right]^{T} .
$$




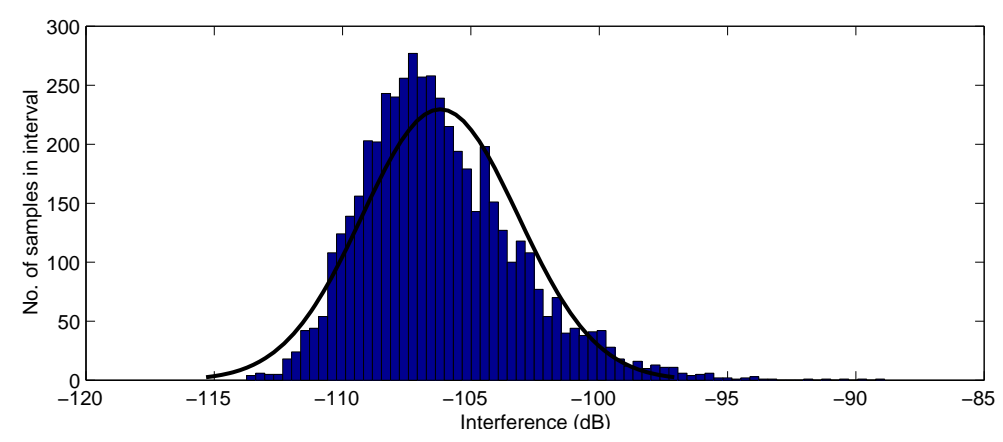

Figure 1: Interference distribution in a random frequency hopping network.

\section{$2.3 \quad$ Measurements}

As argued in the previous section, the interference is characterized by its mean, $m_{I}$, and standard deviation, $\sigma_{I}$. These will affect the outcome of the measurements together with the carrier power, $C_{i}=p_{i}+g_{i i}$. The measurements can be modeled as probability functions depending on these parameters.

$$
h_{i}=h_{i}\left(C, m_{I}, \sigma_{I}\right), \quad i=1,2 .
$$

In GSM the measurement reports consist of RXLEV and RXQUAL [11]. RXLEV is a signal strength measure, which has been quantized in 64 levels, and RXQUAL is a logarithmic measure of the Bit Error Rate (BER), quantized in 8 levels. In general it is not possible to get simple analytical expressions for these functions. However, point-mass approximations can be obtained from simulations for each point in a grid covering the interesting parameter space. Given a point (i.e. a set of parameter values), $C$ - and $I$-sequences can be generated, from which the measurement report can be formed using models of the modulation and coding. Monte-Carlo simulations yield point-mass approximations of the probability functions as exemplified in Figure 2. The corresponding procedure can be applied when forming the probability function of RXLEV.

\section{Maximum Likelihood Estimation}

The estimator can be constructed in numerous ways, but we have chosen a Maximum Likelihood (ML) estimator $[8,9]$, since it successfully enables data fusion and is an implementationally simple algorithm.

The method of ML estimation is based on a simple idea. Different probability density functions generate different data samples and any given data sample is more likely to have come from a particular distribution than from others. The basic ideas will be illustrated by the following example. 


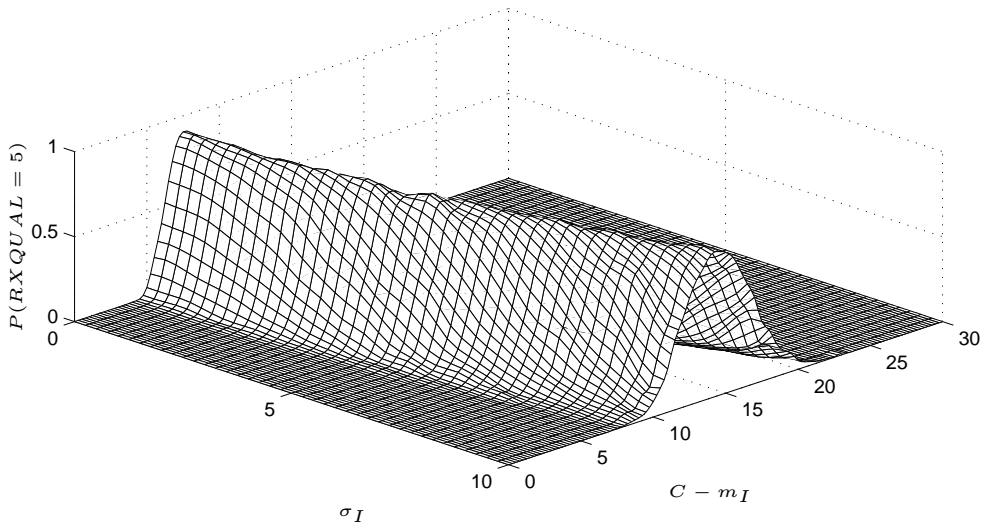

Figure 2: The probability that RXQUAL $=5$ is measured for different values of $C, m_{I}$ and $\sigma_{I}$.

\subsection{A Simple Example}

Consider an exponentially distributed stochastic variable $\mathrm{X}$, parameterized by $\theta$

$$
X \in \operatorname{Exp}(\theta), f_{X}(x ; \theta)=\frac{1}{\theta} e^{-x / \theta}
$$

Let the true value be $\theta=5$. Assume that a measurement $x_{1}=4.3$ has been observed. In order to estimate $\theta$, we form the likelihood function

$$
l_{1}(\theta)=f_{X}\left(x_{1} ; \theta\right)=\frac{1}{\theta} e^{-x_{1} / \theta}
$$

computed for a number of grid points covering the interesting values of $\theta$, see Figure 3a. The natural $\theta$-estimate is the value maximizing this function. When observing a second measurement, the procedure could be repeated analogously as in Figure 3b. Even better is to utilize both measurements using the joint likelihood function, defined as the product of the two likelihood functions. The improvements of the estimate are illustrated in Figures 3c-d. Numerically, it may be more appealing to use logarithmic values. Then the joint likelihood can be defined as

$$
\log l_{t}(\theta)=\sum_{i=1}^{t} \log f_{X}\left(x_{i} ; \theta\right)
$$

\subsection{Adaptive Estimation}

This far a fixed parameter has been assumed. In a real situation, however, the parameter may be time-varying, and therefore adaptivity is important. This is introduced by employing exponential forgetting of the joint likelihood function, which can be implemented by the following recursion

$$
\log l_{t}(\theta)=\log f_{X}\left(x_{t} ; \theta\right)+\lambda \log l_{t-1}(\theta) .
$$


a. $f\left(x_{1} ; \theta\right)$

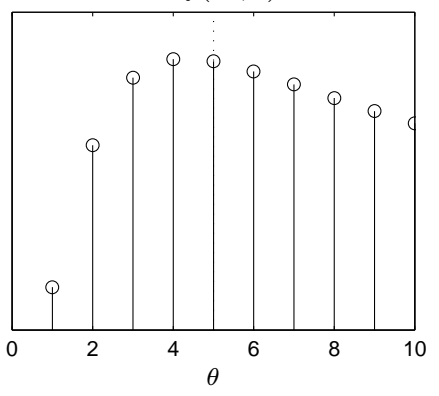

c. $f\left(x_{1} ; \theta\right) f\left(x_{2} ; \theta\right)$

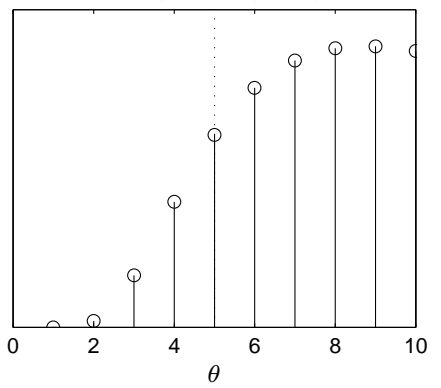

b. $f\left(x_{2} ; \theta\right)$

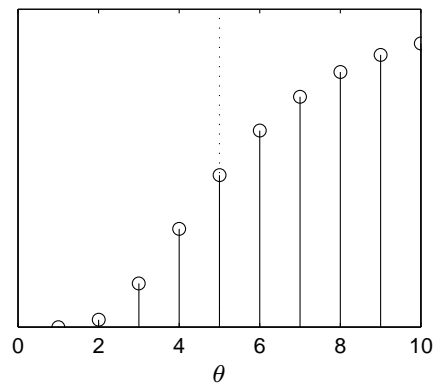

d. $f\left(x_{1} ; \theta\right) \cdot \ldots \cdot f\left(x_{5} ; \theta\right)$

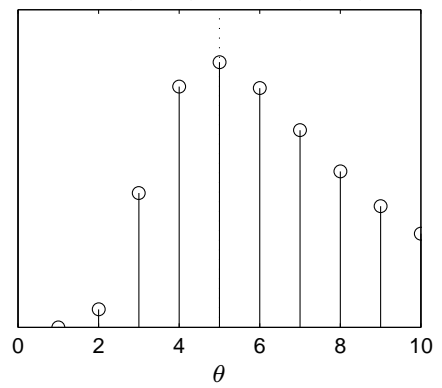

Figure 3: The different likelihood functions

The estimate is then obtained as

$$
\hat{\theta}^{M L}(t)=\underset{\theta}{\arg \max } l_{t}(\theta) .
$$

When there are several parameters to be estimated and several measurements available, all of the above apply. The measurement at time $t, x_{t}$, and the parameter $\theta$ are now vectors. A fundamental result from statistics states that when the measurements are independent, the joint probability function is obtained as the product of the probability functions of each of the measurements.

$$
f_{X}\left(x_{t}, \boldsymbol{\theta}\right)=\prod_{i=1}^{M} f_{X_{i}}\left(x_{t}^{(i)}, \boldsymbol{\theta}\right) .
$$

If the probability function is (close to) zero at some grid points for a certain measurement, the value of the likelihood function will remain (for a while close to) zero at those points. Consequently, the likelihood function is blocked from growing at those points, inhibiting the adaptivity to varying parameters. A solution is to use a threshold value, $f_{m i n}$, and when updating the likelihood use the probability function

$$
g_{X}\left(x_{i}, \theta\right)=\max \left\{f_{X}\left(x_{i}, \theta\right), f_{\min }\right\} .
$$

Finally, the parameters may be changing at different rates. This is solved by post-filtering the estimates using exponential filtering $[3,5]$. with different 
forgetting factors $\mu_{k}$

$$
\hat{\theta}_{k}(t+1)=\left(1-\mu_{k}\right) \hat{\theta}_{k}^{M L}(t+1)+\mu_{k} \hat{\theta}_{k}(t) .
$$

Thus, we propose the following estimation algorithm:

\section{Algorithm: ML Estimation}

Let the measurements at time $t$, be given by the vector $\boldsymbol{x}_{t}$, with the corresponding probability function $f_{X}\left(\boldsymbol{x}_{t} ; \boldsymbol{\theta}\right)$. Define $g_{X}\left(\boldsymbol{x}_{t} ; \boldsymbol{\theta}\right)$ by

$$
g_{X}\left(\boldsymbol{x}_{t} ; \boldsymbol{\theta}\right)=\max \left\{f_{X}\left(\boldsymbol{x}_{t} ; \boldsymbol{\theta}\right), f_{\min }\right\} .
$$

Update the likelihood function according to

$$
\log l_{t}(\boldsymbol{\theta})=(1-\lambda) \log g_{X}\left(\boldsymbol{x}_{t} ; \boldsymbol{\theta}\right)+\lambda \log l_{t-1}(\boldsymbol{\theta}),
$$

with the initial likelihood $l_{0}$ equal to a Gaussian probability density function. The estimate $\boldsymbol{\theta}(t)$ is given by

$$
\hat{\boldsymbol{\theta}}^{M L}(t)=\underset{\boldsymbol{\theta}}{\arg \max } l_{t}(\boldsymbol{\theta}) .
$$

Post-filter the estimates separately as

$$
\hat{\theta}_{i}(t+1)=\left(1-\mu_{i}\right) \hat{\theta}_{i}^{M L}(t+1)+\mu_{i} \hat{\theta}_{i}(t)
$$

\section{Simulations}

The simulation environment is GSM specific and the measurements are available as measurement reports as in the real system. The simulator parameters are summarized in Table 1. In order to get a feel for ML estimation, we first consider a simple case. All parameters are fixed except $m_{I}$ which changes abruptly, due to new mobiles admitted into the system. In this case, the simulator is only used to generate a sequence of measurement reports, which are fed to the estimator. The results are found in Figure 4, where we see that the estimator is able to track the parameters. The operation of the estimator in this case is further illuminated by Figure 5 .

The normal situation is that the carrier $C$ and mean interference $m_{I}$ are subject to fast variations due to shadow and multipath fading, see [12]. The measurement reports in Figure 6 are obtained from network simulations. These are fed to the estimator and the estimated parameters are compared to the true values, see Figure 7. Despite the loss of information in the quantization, the estimates are reasonably accurate. In the example in Figure 7, we obtain the following Root Mean Squared Errors (RMSE): $\operatorname{RMSE}\left\{m_{I}-\hat{m}_{I}\right\}=2.0 \mathrm{~dB}$ and $\operatorname{RMSE}\{\gamma-\hat{\gamma}\}=2.1 \mathrm{~dB}$. 


\begin{tabular}{ll}
\hline Frequency band & $900 \mathrm{MHz}$ \\
Antennas & Sectorized \\
Cell radius & $1000 \mathrm{~m}$ \\
Cell layout & $5 \times 5$ clusters \\
& of 9 cells \\
Frequency hopping & Pseudo-random \\
Control sample interval & $T_{c}=0.48 \mathrm{~s}$ \\
Burst time & $0.577 \mathrm{~ms}$ \\
Mobile station & GSM class 4 \\
Mean mobile station speed & $50 \mathrm{~km} / \mathrm{h}$ \\
\hline
\end{tabular}

Table 1: System simulation parameters.

\section{Conclusions}

In this work we have focused on estimation in cellular radio systems based only on the signals that are readily available. Previous work have demonstrated very good performance relying on analog measurement. In a real system most of the information is lost due to quantization and sampling at a rate that might be as low as $2 \mathrm{~Hz}$ (GSM case). Therefore a different approach is required and for that matter a Maximum Likelihood Estimator has been designed and exemplified in the case of GSM.

The needed probability functions of the measurements cannot be described analytically. Instead point-mass approximations can be obtained from MonteCarlo simulations for each point in a grid covering the interesting parameter space. Using different forgetting factors, the proposed algorithm can be tuned to track parameters varying at different rate individually. Simulations indicate good performance both when the parameters are varying slowly, and when subject to fast variations as in realistic cases.

The algorithm is ready for implementation in a second generation wireless system. The only component that needs to be updated is the software in the base stations, where the output powers are computed. However, this estimation method is general, and will be useful in a third generation wireless system as well. 
a.

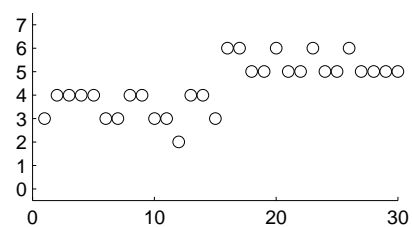

c.

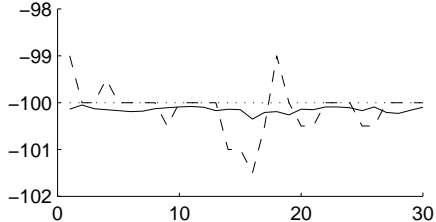

e.

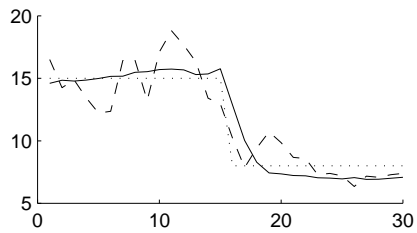

b.

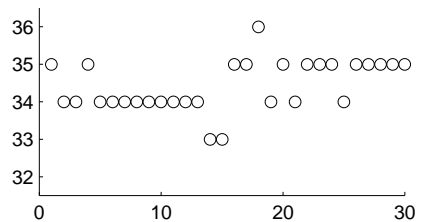

d.

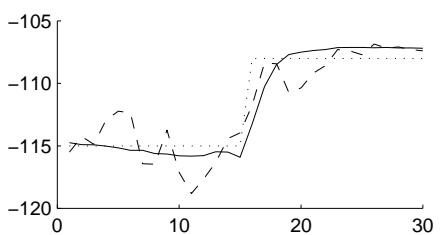

f.

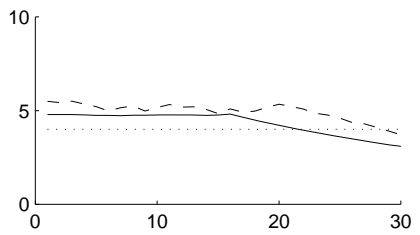

Figure 4: Estimation when there is an abrupt change in the true $m_{I}$. Above are the estimated values before (dashed) and after (solid) post-filtering and the true values (dotted). Measurement reports: a) RXQUAL and b) RXLEV. Estimated parameters: c) Carrier, $C$, d) Mean interference, $m_{I}$, e) $\left.\mathrm{C} / \mathrm{I},\left(C-m_{I}\right), \mathrm{f}\right)$ Interference standard deviation, $\sigma_{I}$.
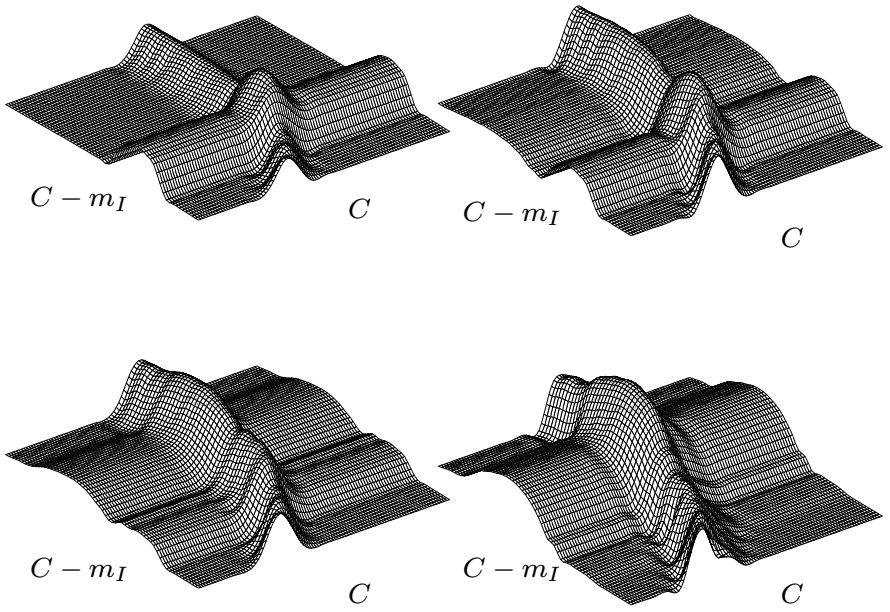

Figure 5: The shape and peak position of the likelihood function changes, when the value of $m_{I}$ changed abruptly. The maximum of this function is seen to move in the $\left(C-m_{I}\right)$-direction. The $\sigma$ direction has been eliminated by selecting the true value. 
a.

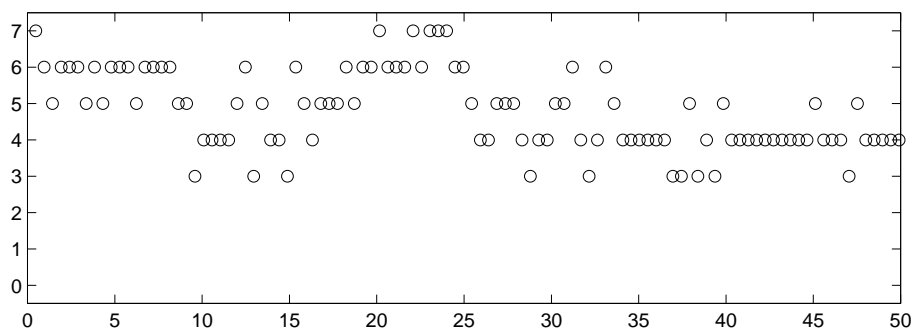

b.

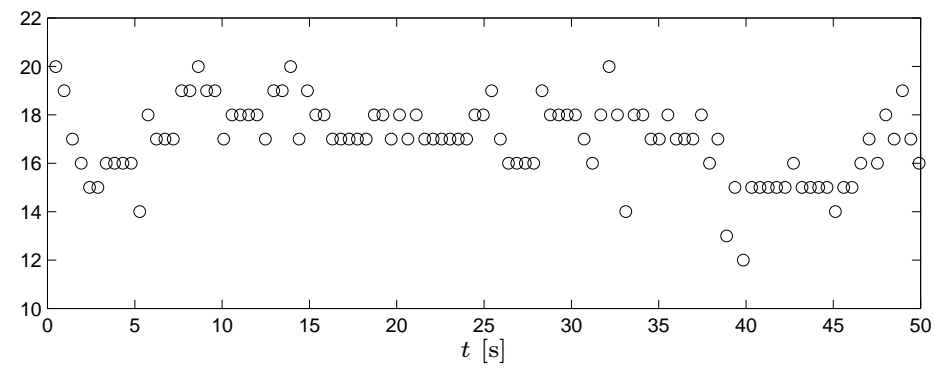

Figure 6: Measurement reports, consisting of a) RXQUAL and b) RXLEV, describing the perceived quality and signal strength respectively. These values are used as input to the proposed algorithm.

a)

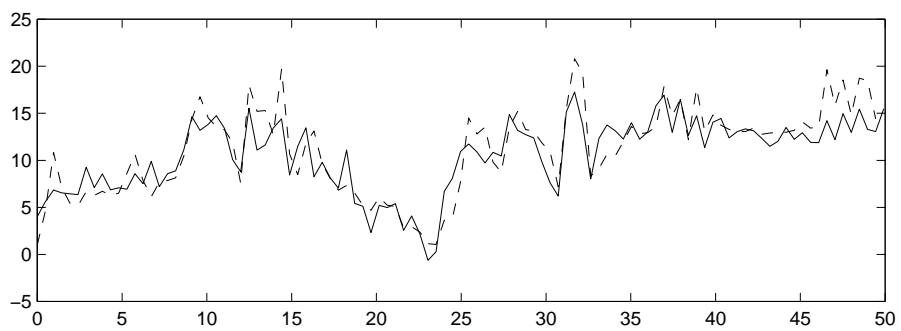

b)

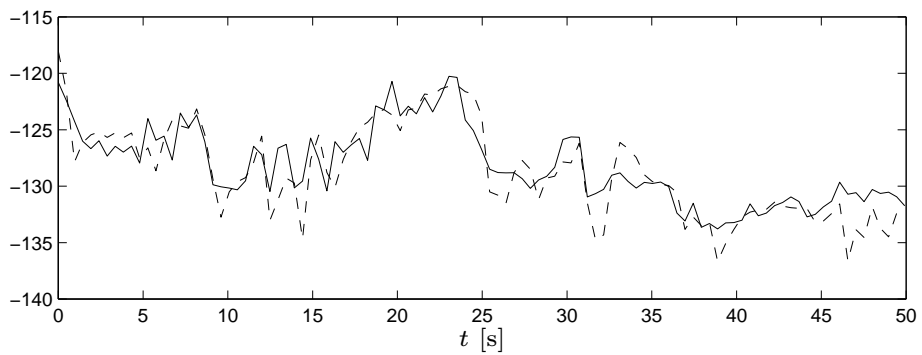

Figure 7: Given the measurement reports in Figure 6 the proposed algorithm extracts e.g. a) $\mathrm{C} / \mathrm{I}$ and b) mean interference, $m_{I}$. The estimated values (solid) are compared to the true ones (dashed). 


\section{References}

[1] M. Almgren, H. Andersson, and K. Wallstedt. Power control in a cellular system. In Proc. IEEE Vehicular Technology Conference, Stockholm, Sweden, June 1994.

[2] M. Andersin, N. B. Mandayam, and D. Y. Yates. Subspace based estimation of the signal to interference ratio for TDMA cellular systems. Wireless Networks, 4(3), 1998.

[3] J. Blom and F. Gunnarsson. Power Control in Cellular Radio Systems. Licenciate Thesis, Linköpings universitet, Sweden, June 1998. Available at http://www. control.isy.liu.se.

[4] F. Gunnarsson, J. Blom, and F. Gustafsson. Method and system for quality-based power control in cellular communications systems. US Patent Application No. 09/031,635, 1998.

[5] F. Gunnarsson, J. Blom, and F. Gustafsson. Power control in cellular systems subject to constraints and time delays. In Proc. IEEE Global Telecommunications Conference, Sydney, Australia, November 1998.

[6] F. Gunnarsson, F. Gustafsson, and J. Blom. Improved performance using nonlinear components in power control algorithms. In Proc. IEEE Vehicular Technology Conference, Houston, TX, USA, May 1999.

[7] F. Gunnarsson, F. Gustafsson, and J. Blom. Pole placement design of power control algorithms. In Proc. IEEE Vehicular Technology Conference, Houston, TX, USA, May 1999.

[8] E.L. Lehmann. Theory of Point Estimation. Statistical/Probability series. Wadsworth \& Brooks/Cole, 1991.

[9] L. Ljung. System Identification - Theory for the User. Prentice Hall, Englewood Cliffs, NJ, USA., 1987.

[10] S. C. Schwartz and Y. S. Yeh. On the distribution function and moments of power sums with log-normal components. The Bell System Technical Journal, 61(7), 1982.

[11] R. Steele. Mobile Radio Communications. Pentech Press, London, England, 1992.

[12] G. L. Stüber. Principles of Mobile Communication. Kluwer Academic Publisher, Boston, MA, USA, 1996.

[13] M. Türkboylari and G.L. Stüber. An efficient algorithm for estimating the signal-to-interference ratio in TDMA cellular systems. IEEE Transactions on Communications, 46(6), 1998. 